\title{
Radio contrast imaging for continuous epidural infusion in humans: a report of three cases
}

This article was published in the following Dove Medical Press journal: Journal of Pain Research

\author{
Takashi Matsusaki \\ Ryuji Kaku \\ Daisuke Ono \\ Arata Taniguchi \\ Hiroshi Morimatsu \\ Department of Anesthesiology and \\ Resuscitology, Okayama University \\ Hospital, Okayama, Japan
}

Correspondence: Takashi Matsusaki Department of Anesthesiology and Resuscitology, Okayama University Graduate School of Medicine, Dentistry, and Pharmaceutical Sciences, 2-5-I Shikata-cho, Kita-ku, Okayama 700-8558, Japan

Tel +81862357778

Fax +8I 862356984

Email matusakik@ybb.ne.jp

\begin{abstract}
There are no reports of human research on continuous epidural contrast injection, and there are no definite methods to investigate the spread of drugs injected continuously into the epidural space. We investigated the feasibility of continuous epidural contrast injection in patients undergoing computed tomography (CT)-guided therapy. In this study, a combination of a contrast agent mixed with $0.75 \%$ ropivacaine was used as the test drug. The main outcome evaluated was the feasibility of continuous epidural contrast imaging by CT scan following epidural injection of a contrast agent with $0.75 \%$ ropivacaine. We studied three patients who underwent CT-guided procedures and found that continuous epidural contrast injection was possible without any deleterious effects, such as an allergic reaction. The spread of the contrast agent was not consistent with the level of the clinical analgesic effect. Continuous epidural contrast injection is a feasible procedure. The results of our study might contribute to future research on continuous epidural contrast administration, as well as provide patients with superior analgesia.
\end{abstract}

Keywords: continuous epidural contrast, 3D-CT scan, spread of epidural analgesia

\section{Introduction}

It is well known that continuous epidural analgesia via a catheter in the back provides effective intra- and postoperative analgesia by blocking the conduction of pain signals at the nerve roots in the spine, which occur in response to invasive stimuli. ${ }^{1}$ However, cases of failed continuous epidural block administered by attending anesthesiologists, because of catheter migration out of the epidural space, have been reported. ${ }^{2}$ In order to secure epidural pain effects, a catheter is usually inserted into the epidural space; however, the correct position of the catheter and spread of analgesics cannot be assured. Traditionally, analgesic effect is confirmed by testing the loss of cold sensation, while catheter placement is guided by assessing loss of resistance to the injection of air or saline. Recently, epidural contrast radiography has been shown to facilitate more accurate placement of the catheter in the correct epidural space as compared to the traditional loss of resistance method. ${ }^{3}$ However, despite accurate catheter placement, prediction of the correct spread of drugs administered for continuous epidural block is challenging because there are several factors influencing the spread of drugs continuously injected into the epidural space. ${ }^{4}$ Additionally, although a previous in vitro study showed that bolus drug injection through a catheter results in more even spread of the drug compared with a continuous infusion, no information from human studies exists regarding how the spread following continuous injection compares with that following bolus injection. ${ }^{5}$ 
Some previous clinical studies involving bolus epidural contrast injection showed that the spread of contrast is consistent with the expected analgesic effects. ${ }^{6-8}$ However, no actual information from contrast radiographic studies on the spread of continuously injected epidural analgesic agents, including the methods used, exists.

The objective of this study was to evaluate the feasibility, safety and extent of spread of a contrast agent given by continuous epidural injection in patients undergoing computed tomography (CT)-guided procedures under epidural anesthesia and to determine the appropriate concentration of the contrast agent to be used for this.

\section{Patients and methods}

This prospective, invasive clinical research was conducted at our tertiary teaching hospital in Japan with institutional review board approval of Okayama University Graduate School of Medicine, Dentistry and Pharmaceutical Sciences and Okayama University Hospital, Ethics Committee (Number: 05002) and after obtaining the patients' written informed consent for study participation. This study complied with the principles of the Declaration of Helsinki.

Patients who required CT-guided procedures, such as embolism of a liver artery or radiofrequency treatment of the liver, which are usually performed under epidural analgesia, were included. Only conscious patients with whom we would be able to communicate were included.

Patients with allergies to contrast agents, renal dysfunction (serum creatinine $>2.0 \mathrm{mg} / \mathrm{dL}$ ), liver dysfunction (serum AST/ALT $>100 \mathrm{mg} / \mathrm{dL}$ ), severe cardiac dysfunction (ejection fraction $<40 \%$ ), convulsions, severe asthma or thyroid dysfunction were excluded from the study.

According to a previous anticancer therapy protocol, the required sample size in this study was three to nine patients. ${ }^{9}$ Since we did not know what dose of epidural contrast would be appropriate for continuous administration, we created a new protocol for this research, keeping the concentration of contrast at the lowest level possible for patient safety.

The main outcome evaluated was the feasibility of continuous epidural contrast administration at the end of the therapeutic procedure being performed under CT guidance by authorized radiologists. The secondary outcome evaluated was the safety of the procedure in terms of the viscosity resulting from mixing the contrast agent with ropivacaine. Additionally, we observed the patients for side effects to the procedure, including allergic and anaphylactic reactions to the injection of contrast. We also investigated the relationship between the level of analgesic effect and epidural spread of the contrast agent.

The study was to be performed in three steps, each step using different concentrations of the contrast agent mixed with a local anesthetic and being performed in three patients. At each step, the corresponding dose of contrast agent was administered epidurally, and the patient was assessed clinically for the local anesthetic effect, while CT scan was performed to assess the spread of the contrast agent.

If Step 1 could not be successfully performed, Step 2 would be performed, and if Step 2 was unsuccessful, Step 3 would be performed. According to the study protocol, if Step 3 could not be successfully performed in even a single case, continuous epidural contrast injection would be deemed impossible.

\section{Study protocol}

Step 1: Continuous epidural injection of $0.75 \%$ ropivacaine $10 \mathrm{~mL}+$ contrast $10 \mathrm{~mL}$ (iohexol) + saline $10 \mathrm{~mL}$ was administered at the rate of 4-8 $\mathrm{mL} / \mathrm{h}$, depending on the patient's pain and vital signs, using a syringe pump. This dose of epidural contrast was considered successful if it resulted in appropriate spread of the contrast agent, assessed by authorized radiologists. If it was unsuccessful, Step 2 was performed in the next patient.

Step 2: Epidural injection of $0.75 \%$ ropivacaine $10 \mathrm{~mL}+$ contrast (iohexol) $15 \mathrm{~mL}+$ saline $5 \mathrm{~mL}$ (continuous injection rate: $4-8 \mathrm{~mL} / \mathrm{h}$, depending on the patient's pain and vital signs).

If Step 2 was unsuccessful, Step 3 was to be performed in the next patient.

Step 3: Epidural injection of $0.75 \%$ ropivacaine $10 \mathrm{~mL}+$ contrast (iohexol) $20 \mathrm{~mL}$ (continuous injection rate: $4-8$ $\mathrm{mL} / \mathrm{h}$, depending on the patient's pain and vital signs). If epidural contrast injection was successful in all three cases at any of the three steps without any adverse events, the research was deemed successful.

\section{Anesthesia procedure}

An epidural catheter was inserted preoperatively in the CT room with the patient in the lateral position. The appropriate insertion site was decided by the attending anesthesiologist and radiologists, depending on the subsequent interventional radiological procedure to be performed. Accurate placement of the needle (Perifix Tuohy epidural needle [BMG332186], 18G×4.75; B Braun Medical Inc., Bethlehem, PA, USA) into the epidural space was confirmed by the traditional method of "loss of resistance" to the injection of saline. After the epidural catheter was inserted via the needle in a cephalad 
direction, 1\% lidocaine with epinephrine (1:50,000; $4 \mathrm{~mL})$ was injected epidurally, taking care to exclude inadvertent intravenous (IV) insertion. Thereafter, the catheter was fixed at a depth of $3-5 \mathrm{~cm}$ from the insertion site using sterile tape.

The patients were given enough volume of $1.5 \%$ lidocaine epidurally to secure analgesia during the procedure. Finally, the level of epidural analgesia was checked by assessing the loss of cold sensation over the blocked area and surgery was commenced.

Thereafter, at the time of commencement of the radiological procedure, continuous epidural infusion of the contrast agent with $0.75 \%$ ropivacaine was also commenced. Analgesic assessment was performed every 10-15 minutes.

\section{Intraoperative pain control protocol}

If the patient felt pain and required further analgesia, analgesics were given according to our protocol for analgesia management, wherein either the dose of the continuous epidural analgesic drug was increased, the surgeon was asked to inject an additional amount of $1 \%$ lidocaine, a bolus of fentanyl $(50-100 \mu \mathrm{g})$ was administered IV or a continuous IV infusion of dexmedetomidine $(0.4-0.8 \mu \mathrm{g} / \mathrm{kg} / \mathrm{hr})$ was administered.

\section{Surgical procedure}

The interventional radiological procedures performed were embolism of the renal artery in two of the cases, which required analgesia at the Th6-10 area, and radiofrequency ablation of a lung tumor in one case, which required analgesia at the Th2-8 level.
After these procedures were completed, a check CT scan from the cervical to lumbar level was performed in order to confirm the feasibility of continuous epidural contrast injection and to rule out complications of the radiological procedures, such as bleeding and pneumothorax.

\section{Results}

Case 1: A 71-year-old man (height $168 \mathrm{~cm}$, body weight $70 \mathrm{~kg}$, body mass index [BMI] $25 \mathrm{~kg} / \mathrm{m}^{2}$ ) had a history of right renal carcinoma with hypertension. Right renal artery embolization was scheduled under epidural anesthesia, which was administered at the Th8/9 level. Before surgery, 1.5\% lidocaine $(6 \mathrm{~mL})$ was administered via the epidural catheter. Fifteen minutes later, the level of anesthesia was confirmed at Th6-10 using the loss of cold sensation test. His vital signs remained stable, and continuous epidural injection (6 $\mathrm{mL} / \mathrm{h}$ ) of the mixture of $0.75 \%$ ropivacaine $10 \mathrm{~mL}+$ contrast (iohexol) $10 \mathrm{~mL}+$ saline $10 \mathrm{~mL}$ was commenced at the same time as renal artery embolization was started by the radiologists. The surgical duration was 1 hour and 50 minutes. Near the end of surgery, the patient complained of back pain, for which we gave him fentanyl $(100 \mu \mathrm{g})$. A total of $10 \mathrm{~mL}$ of the test solution was administered. Continuous epidural contrast injection was possible, and the drug was seen to spread from Th4 to 10 on three dimensional (3D)-CT scan (Figure 1A, B), which was inconsistent with the clinical spread assessed by the cold test (Th6-10).

Case 2: A 59-year-old man (height $166 \mathrm{~cm}$, body weight $64 \mathrm{~kg}$, BMI $23 \mathrm{~kg} / \mathrm{m}^{2}$ ) had a history of left renal carcinoma with hypertension, for which left renal artery embolization

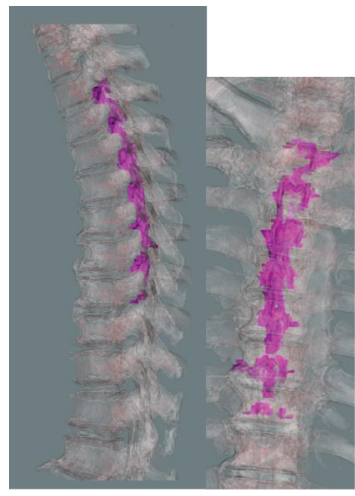

A

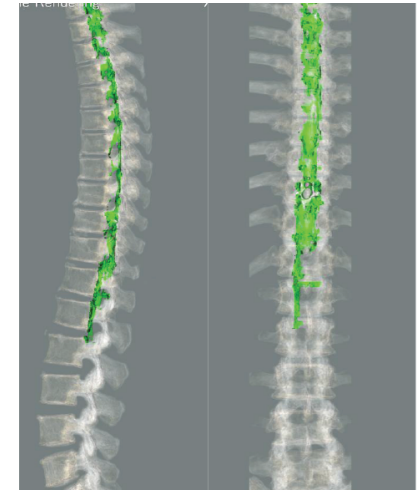

D

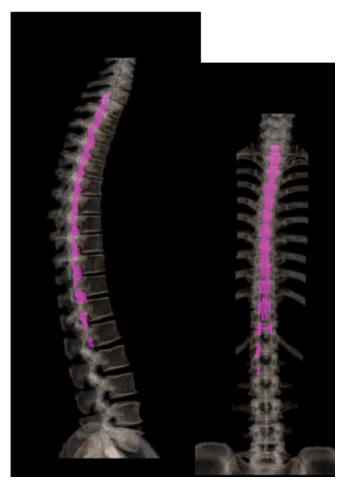

E

$\mathbf{F}$

Figure I 3D-CT in the lateral and posterior views following continuous epidural contrast injection in three cases.

Notes: (A, B) The purple color represents the contrast agent, which spread from Th4 to 10 in case I. (C, D) 3D-CT following continuous epidural contrast injection in case 2. The green color represents the contrast agent, which spread from ThI to L2. (E, F) 3D-CT following continuous epidural contrast injection in case 3. The purple color represents the contrast agent, which spread from $\mathrm{C} 7$ to $\mathrm{Th} / 2$.

Abbreviation: CT, computed tomography. 
was scheduled under epidural anesthesia, the catheter for which was inserted at Th10/11. Before surgery, 1.5\% lidocaine $(6 \mathrm{~mL})$ was given via the epidural catheter. Twenty minutes later, the anesthetized area was confirmed over Th6-10 by the cold test. His vital signs were stable and continuous epidural injection of the local anesthetic agent $(0.75 \%$ ropivacaine $10 \mathrm{~mL}+$ contrast (iohexol) $10 \mathrm{~mL}+$ saline $10 \mathrm{~mL}$ ) at the rate of $6 \mathrm{~mL} / \mathrm{h}$ was commenced at the same time as the radiologists started the renal artery embolization procedure (6 $\mathrm{mL} / \mathrm{h})$. Surgical duration was 2 hours and 10 minutes, during which he did not complain of any pain. We used a total of 13 $\mathrm{mL}$ of the test contrast-anesthetic combination. Continuous epidural contrast injection was possible and the drug spread from Th1 to L2 (Figure 1C, D), as shown on 3D-CT scan, which was inconsistent with the clinical anesthetic effect (Th6-10), as assessed by the cold test.

Case 3: A 52-year-old man (height $168 \mathrm{~cm}$, body weight $67 \mathrm{~kg}$, BMI $24 \mathrm{~kg} / \mathrm{m}^{2}$ ) had a metastatic carcinoma of the left lung with no other significant medical history. He was scheduled for radiofrequency ablation (RFA) of the lung tumor under epidural anesthesia, for which the catheter was inserted at the Th5/6 level. Before surgery, 1.5\% lidocaine (6 $\mathrm{mL})$ was administered epidurally. Twenty minutes later, the anesthetized area was confirmed over Th3 -8 by the cold test. His vital signs were stable, and continuous epidural injection $(0.75 \%$ ropivacaine $10 \mathrm{~mL}+$ contrast (iohexol) $10 \mathrm{~mL}+$ saline $10 \mathrm{~mL}$ ) at the rate of $5 \mathrm{~mL} / \mathrm{h}$ was commenced at the same time as RFA of the lung tumor. Surgical duration was 2 hours and 25 minutes. He complained of left shoulder pain during surgical manipulation, which was controlled by fentanyl 100 $\mu \mathrm{g}$. We used a total of $12 \mathrm{~mL}$ of the test contrast/anesthetic combination. Continuous epidural contrast injection was possible and showed a radiological spread from $\mathrm{C} 7$ to Th12 on 3D-CT scan (Figure 1E, F), which was inconsistent with the clinical spread determined by the cold test (Th2-8).

None of the patients experienced any anaphylactic reactions or delayed complications, such as renal or hepatic damage due to the test drugs (Table 1).

Step 1 was successful in all three patients and Steps 2 and 3 were not required for any of them.

\section{Discussion}

In this study, continuous epidural contrast injection was possible without any deleterious effects. The concentration of epidural contrast used (the lowest of the concentrations that was to be tested in this study) allowed adequate visualization of drug spread. Spread of the epidural contrast agent was not
Table I Summary of the three cases

\begin{tabular}{|l|l|l|l|}
\hline & Case I & Case 2 & Case 3 \\
\hline Epidural insertion level & Th8/9 & ThI0/II & Th5/6 \\
\hline Total dose of test drug $(\mathrm{mL})$ & 10 & 13 & I2 \\
\hline Injection rate $(\mathrm{mL} / \mathrm{h})$ & 6 & 6 & 5 \\
\hline Anesthesia level $^{\mathbf{a}}$ & & & \\
\hline Before surgery & Th6-10 & Th6-10 & Th3-8 \\
\hline After surgery & Th6-10 & Th6-10 & Th2-8 \\
\hline Spread of contrast on 3D-CT & Th4-10 & ThI-L2 & C7-Th/2 \\
\hline Pain during surgery & + & - & + \\
\hline Adverse effects & - & - & - \\
\hline
\end{tabular}

Note: ${ }^{2}$ Anesthesia level was assessed by testing for loss of cold sensation.

Abbreviation: $\mathrm{CT}$, computed tomography.

consistent with the clinical analgesic effect, as assessed by the cold test.

The safety of continuous contrast injection into the epidural space is not known, although previous literature has evaluated the safety of bolus administration of epidural contrast (epidurography). ${ }^{6-8}$ Although we anticipated anaphylactic reactions and delayed adverse effects, such as hepatic and renal dysfunction, none of our patients experienced these effects.

Traditionally, a standard epidurogram is used for the differential diagnosis of suspected disorders of the epidural space, although CT scan is also an effective method to accurately confirm the anatomy of the epidural space. ${ }^{10}$ Hence, we conducted this study on patients who required their surgery to be performed under CT guidance.

In this study, we were concerned that the high viscosity of the contrast agent/anesthetic mixture would lead to obstruction of the epidural catheter. Hence, before conducting this study, we performed an in vitro experiment to assess this, using all three concentrations of the test mixture (injection speed: $4-6 \mathrm{~mL} / \mathrm{h}$ ), which showed that there was no obstruction of the epidural tube by the test mixture.

A previous study in a porcine model demonstrated that the spread of an injection of epidural dye solution is more extensive after a single bolus compared with continuous infusion of the solution (spreading to a distance of $8.9 \pm 2.6 \mathrm{~cm}$ in the continuous group compared with $15.2 \pm 2.7 \mathrm{~cm}$ in the bolus group; $P<0.001) .{ }^{11}$ Another study also examined the spread of contrast medium containing $1 \%$ new methylene blue dye in the epidural space of recumbent dogs. ${ }^{12}$ However, there are no previous studies on epidural dye injection in human subjects. Hence, we chose to inject a contrast agent rather than a dye or other medicine in this study. We assessed the spread of continuous epidural contrast using 3D-CT in humans. Since 
there is no established method for this procedure, including the appropriate concentration of the contrast medium to be used, we injected the minimal dose we believed would be both safe and effective. We believe our results will contribute to future studies on this topic.

We decided our study protocol after confirming its safety in patients on anticancer drug therapy (Phase I study), to determine whether continuous epidural contrast injection performed by specialized radiologists would be possible. We started the assessments with the minimum concentration of contrast in order to minimize the adverse effects of contrast injection. In our study, the lowest concentration of the mixture (saline, contrast and ropivacaine) evaluated was found to give adequate results.

We also found that epidural spread of the continuously administered contrast agent was not consistent with the observed clinical effect (the spread of contrast was more extensive than the anesthetized area assessed by the cold test and pain). In previous literature, the clinical analgesic effect of bolus injection of the test mixture was similar to the area of spread of the contrast. However, with continuous epidural contrast injection, the spread of the contrast agent was greater than that suggested by the clinical analgesic effects.

Several factors could explain the inconsistency between extension of the contrast agent and analgesic effects. The distribution of contrast may not have been uniform. A previous study suggested that the factors influencing epidural spread of an injected substance include insertion site of the epidural needle (thoracic or lumbar), the total amount of anesthetic injected, patient factors (age and position) and the anesthesiologist's technique. ${ }^{13}$ Hence, all these factors need to be considered when interpreting our results. Metabolism of the contrast agent and nonuniform spread of the agent in the epidural space could be two possible reasons for the inconsistency between clinical effect and radiological spread of the contrast agent. "Compartmentalization" of the epidural space could also be another reason for the inconsistency between clinical effects and radiological spread of the test drug in our study. Savolaine et al investigated the lumbar epidural space in 40 patients by CT scan to evaluate degenerative disc disease, which showed that division of the anterior and posterior epidural space was complex. ${ }^{14}$ As shown in Figure 2, the spread of epidural contrast in our cases was not uniform, and this nonuniformity could partly explain the inconsistency between extension of the contrast agent and analgesic effects.

Our study has some limitations. First, the number of subjects in our study was too small to assess the safety and feasibility of our protocol. Second, we were not able to adequately explain the inconsistency between the analgesic effect and the spread of contrast. Third, we did not consider other individual factors, such as deformity of the spine and physical condition, that would affect the spread of contrast agent in the epidural space.

\section{Conclusion}

Continuous epidural contrast injection can be performed without any deleterious consequences. The concentration of our contrast agent mixture was found to be adequate for visualization of the epidural space using 3D-CT scan. The spread of continuously administered epidural contrast is not consistent with clinical effects, such as analgesia, with the drug spreading over a wider area than that indicated by the clinical level of the block. Our results might not only contribute to future research regarding continuous epidural injections, but also provide patients with superior analgesia. Further investigations in a larger case series are needed to confirm our results.
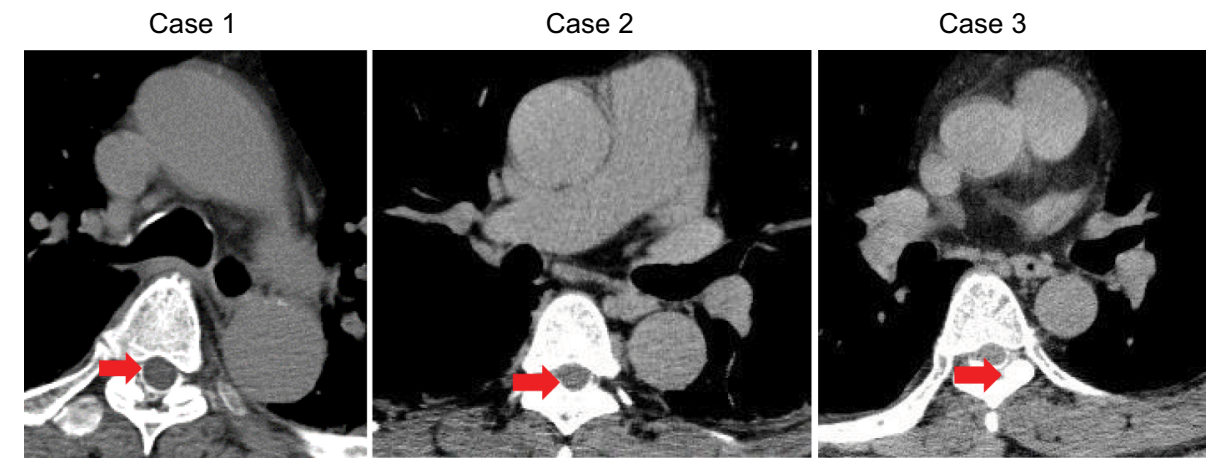

Figure 2 Axial CT views in the three cases.

Notes: Red line shows epidural space. The white area around the spinal cord shows that the spread of contrast was not uniform, since the contrast agent did not spread to anterior parts of the epidural space, spreading only to the posterior space.

Abbreviation: CT, computed tomography. 


\section{Acknowledgments}

We thank Aya Ono, MD, PhD and Shiro Hinotsu, MD, PhD (New Clinical Development of Okayama University Hospital) for their assistance in the design of our research. We would not have been able to complete this research without the radiological support of Takahito Hiraki, MD, PhD. We also really appreciate Yuko Mihara (research nurse). Financial support for this work was provided solely by the institutions and departments named in the author affiliations.

\section{Disclosure}

The authors report no conflicts of interest in this work.

\section{References}

1. Flisberg P, Rudin A, Linnér R, Lundberg CJF. Pain relief and safety after major surgery a prospective study of epidural and intravenous analgesia in 2696 patients. Acta Anaesthesiol Scand. 2003;47(4):457-465.

2. Shafer AL, Donnelly AJ. Management of postoperative pain by continuous epidural infusion of analgesics. Clin Pharm. 1991;10(10):745-764.

3. Ghia Jn, Arora Sk, Castillo M, Mukherji Sk. Confirmation of location of epidural catheters by epidural pressure waveform and computed tomography cathetergram. Reg Anesth Pain Med. 2001;26(4):337-341.

4. Shaparin N, Bernstein J, White RS, Kaufman A. Bevel direction of epidural needles reliably predicts direction of catheter placement and contrast spread in human cadavers: results of a pilot study. J Clin Anesth. 2014;26(8):587-590.
5. Kaynar AM, Shankar KB. Epidural infusion: continuous or bolus? Anesth Analg. 1999;89(2):534.

6. Hogan Q. Epidural catheter tip position and distribution of injectate evaluated by computed tomography. Anesthesiology. 1999;90(4):964-970.

7. Yokoyama M, Hanazaki M, Fujii H, et al. Correlation between the distribution of contrast medium and the extent of blockade during epidural anesthesia. Anesthesiology. 2004;100(6):1504-1510.

8. Afshan G, Chohan U, Khan FA, Chaudhry N, Khan ZE, Khan AA. Appropriate length of epidural catheter in the epidural space for postoperative analgesia: evaluation by epidurography. Anaesthesia. 2011;66(10):913-918.

9. Newell DR, Silvester J, McDowell C, Burtles SS; Cancer Research UK. The Cancer Research UK experience of pre-clinical toxicology studies to support early clinical trials with novel cancer therapies. Eur J Cancer. 2004;40(6):899-906.

10. Fukushige T, Kano T, Sano T, Irie M. Computed tomographic epidurography: an aid to understanding deformation of the lumbar dural sac by epidural injections. Eur J Anaesthesiol. 1999;16(9):628-633.

11. Mowat I, Tang R, Vaghadia H, et al. Epidural distribution of dye administered via an epidural catheter in a porcine model. Br JAnaesth. 2016;116(2):277-281.

12. Son W-G, Kim J, Seo J-P, et al. Cranial epidural spread of contrast medium and new methylene blue dye in sternally recumbent anaesthetized dogs. Vet Anaesth Analg. 2011;38(5):510-515.

13. Visser WA, Lee RA, Gielen MJ. Factors affecting the distribution of neural blockade by local anesthetics in epidural anesthesia and a comparison of lumbar versus thoracic epidural anesthesia. Anesth Analg. 2008;107(2):708-721.

14. Savolaine ER, Pandya JB, Greenblatt SH, Conover SR. Anatomy of the human lumbar epidural space: new insights using CT-epidurography. Anesthesiology. 1988;68(2):217-220.
Journal of Pain Research

\section{Publish your work in this journal}

The Journal of Pain Research is an international, peer reviewed, open access, online journal that welcomes laboratory and clinical findings in the fields of pain research and the prevention and management of pain. Original research, reviews, symposium reports, hypothesis formation and commentaries are all considered for publication.

\section{Dovepress}

The manuscript management system is completely online and includes a very quick and fair peer-review system, which is all easy to use. Visit http://www.dovepress.com/testimonials.php to read real quotes from published authors. 